\title{
GLR-1, a Non-NMDA Glutamate Receptor Homolog, Is Critical for Long-Term Memory in Caenorhabditis elegans
}

\author{
Jacqueline K. Rose, Karla R. Kaun, Sylvia H. Chen, and Catharine H. Rankin \\ Department of Psychology and Brain Research Centre, University of British Columbia, Vancouver, British Columbia, Canada, V6T 1Z4
}

\begin{abstract}
Long-term memory for habituation to tap in Caenorhabditis elegans depends on glr-1, a homolog of mammalian non-NMDA glutamate receptors; mutations in $g l r-1$ blocked long-term memory formation. Green fluorescent protein (GFP) constructs were used to visualize glr-1 expression in the interneurons of the mechanosensory circuit and synaptobrevin in the tap sensory neurons of trained and untrained worms. Trained animals had less GLR-1::GFP expression than untrained animals; there was no difference in the vesicle marker synaptobrevin. Heat shock during training blocked both the behavioral expression of long-term memory and the change in GLR-1::GFP expression. Thus, long-term memory in C. elegans is dependent on $g l r-1$ and likely involves changes in the expression or localization of glutamate receptors.
\end{abstract}

Key words: C. elegans; habituation; long-term memory; glutamate receptors; synaptobrevin; LTD

\section{Introduction}

Glutamate receptor trafficking has been hypothesized as a mechanism of plasticity in the mammalian brain (for review, see Lüscher and Frerking, 2001; Malinow and Malenka, 2002). Stimuli that produce long-term potentiation produce an increase in AMPA-type glutamate receptors inserted into neuronal membranes, whereas stimulation that produces long-term depression (LTD) produces a decrease in the AMPA receptors. Thus far, receptor trafficking as a result of learning has not been shown in any preparation. In this paper, we report that glutamate receptors are critical for long-term memory for habituation in Caenorhabditis elegans and that glutamate receptor expression is altered as a function of training.

C. elegans shows protein synthesis-dependent retention of habituation of the tap withdrawal response $24 \mathrm{hr}$ after distributed training (Beck and Rankin 1995, 1997; Rose et al., 2002). The primary elements of the neural circuit for tap are five sensory neurons and four pairs of interneurons (as well as a large number of motor neurons) (Chalfie et al., 1985; Wicks and Rankin, 1995). The tap response is mediated by electrical connections and modulated by chemical connections (Chalfie et al., 1985; Wicks and Rankin, 1995). The gene EAT-4 encodes a vesicular glutamate transporter and is expressed in the tap circuit sensory neurons (ALMs, AVMs, and PLMs) (Avery 1993; Bellocchio et al., 1998; Lee et al., 1999). Mutation of EAT-4 alters the expression of longterm memory (LTM) for habituation (Rose et al., 2002). When a

Received June 25, 2003; revised Sept. 3, 2003; accepted Sept. 3, 2003.

This work was funded by a Natural Sciences and Engineering Research Council of Canada (NSERC) operating grant (C.H.R.), an NSERC postgraduate scholarship B (J.K.R.), and Michael Smith Foundation for Health Research and Canadian Institutes of Health Research fellowships (J.K.R.). We thank Amelia Wan and Stephan Steidl for scoring data and Lindsay Arscott and Mark McConkey for data on response to tap and trains.

Correspondence should be addressed to Catharine H. Rankin, Department of Psychology and Brain Research Centre, University of British Columbia, 2136 West Mall, Kennedy Building, Vancouver, British Columbia, Canada, V6T 1Z4. E-mail: crankin@psych.ubc.ca.

Copyright $\odot 2003$ Society for Neuroscience $\quad$ 0270-6474/03/239595-05\$15.00/0 tap stimulus was used for training, eat-4(ky5) worms did not exhibit LTM for habituation; however, if trained with a stronger stimulus, a train of taps, LTM was observed. From this, we hypothesize that, with a strong enough stimulus, sufficient glutamate is released from the mechanosensory neurons to produce LTM for habituation.

The necessity of presynaptic glutamate release suggests that postsynaptic glutamate receptors play an important role in LTM for habituation. GLR-1 (glutamate receptor 1) encodes a receptor subunit of a non-NMDA excitatory ionotropic glutamate receptor subtype; it has $40 \%$ homology with mammalian AMPA receptors. The two glr-1 alleles identified in C. elegans are expressed on the tap circuit interneurons (AVAs, AVBs, AVDs, and PVCs). $g l r-1(n 2461)$ is a deletion mutation, and $g l r-1(k y 176)$ is a loss-offunction mutation produced by a premature stop codon (Hart et al., 1995; Maricq et al., 1995).

To investigate the role of $g l r-1$ receptors in LTM for habituation training in C. elegans, wild-type (N2), glr-1(n2461), glr1(ky176), and N2 worms treated with an AMPA receptor antagonist were given distributed habituation training and were tested for LTM. In addition, the effects of LTM training on the genes expressed in the tap sensory neurons and command interneurons were examined using two green fluorescent protein (GFP) constructs (Nonet, 1999; Rongo and Kaplan, 1999).

Parts of this work have previously been published in abstract form (Rose et al., 2001).

\section{Materials and Methods}

Animals. Worms were maintained on nematode growth medium (NGM) agar seeded with Escherichia coli (OP50) (Brenner, 1974). N2 C. elegans Bristol (N2) and $g l r-1(n 2461)$ were obtained from the Caenorhabditis Genetics Center (University of Minnesota, Minneapolis, MN), glr1(ky176) was from V. Maricq (University of Utah, Salt Lake City, UT), GLR-1::GFP was from J. Kaplan (Harvard University, Boston, MA), and pMEC-7::SNB-1::GFP was from M. Nonet (Washington University, St. Louis, MO). 


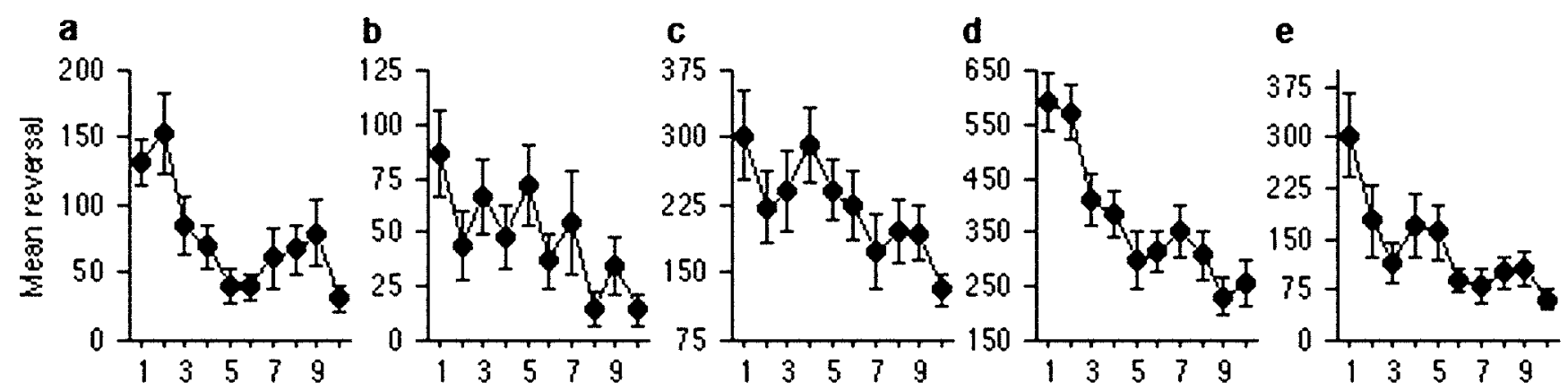

Figure 1. Mutations in glr-1 do not impair short-term habituation at a $60 \mathrm{sec}$ ISI. Mean response magnitude in pixels for wild-type ( $a$ ) and $n 2461$ ( $b$ ) worms given 20 taps at a 60 sec ISI (ky 176 does not respond to taps). Data are presented averaged into 10 bins of two responses each. Both groups show a similar gradual decrease in response magnitude with repeated stimulation. Mean response magnitude in pixels for wild-type $(c), n 2461(d)$, and ky $176(e)$ worms given 20 trains of taps ( 1 train is equivalent to 6 taps within $600 \mathrm{msec}$ ) at a 60 sec ISI. Data are presented averaged into 10 bins of two responses each, as above. All groups show a similar gradual decrease in response magnitude with repeated stimulation. Paired comparisons between bin 1 (average response to the first two stimuli) and bin 10 (average response across the last two stimuli) were significant in all cases with adjustment for multiple comparisons (N2 taps, $t_{(19)}=4.73, p<0.01 ; n 2461$ taps, $t_{(19)}=3.21, p<0.05 ;$ N2 trains, $t_{(17)}=3.82, p<0.05 ; n 2461$ trains, $t_{(18)}=5.11, p<0.01 ; k y 176$ trains, $\left.t_{(15)}=4.33, p<0.01\right)$.

Behavioral procedures. Habituation stimuli (taps-trains) were delivered using a Grass Instruments (Quincy, MA) S88 stimulator driving a mechanical tapper exerting 1-2 $\mathrm{N}$ of force to the side of the Petri plate. For studies of short-term habituation, 20 single tap or train (train is six taps in $600 \mathrm{msec}$ ) stimuli were delivered to single worms on blank NGM agar plates at a $60 \mathrm{sec}$ interstimulus interval (ISI). For studies of LTM for habituation groups of 10-153-d-old worms were placed on E. coli seeded Petri plates $12-18 \mathrm{hr}$ before training. On the training day, experimental worms were given distributed habituation training (four blocks of 20 taps or trains at a $60 \mathrm{sec}$ ISI separated by $1 \mathrm{hr}$ rest periods); control worms were given a single tap or train. One hour after training, all worms were transferred to individual E. coli streaked plates. During testing ( $24-28 \mathrm{hr}$ after training), each worm received 10 taps or trains (whichever was the same as that worm's training) at $60 \mathrm{sec}$ ISI. The responses to the 10 test taps were scored and used in the data analysis. In studies in which heat shock was used, plates were sealed with Parafilm and submerged in a water bath at $32^{\circ} \mathrm{C}$ for 40 min during the rest period between training blocks. Sham heat shock involved submerging plates in a water bath at $21^{\circ} \mathrm{C}$ in the same procedure.

For studies on the role of 6,7-dinitroquinoxaline-2,3(1 H,4 H)-dione (DNQX) on LTM, the drug was dissolved in $35 \mathrm{~mm} \mathrm{NaOH}$ to prepare 10 mM DNQX. Pilot experiments showed no differences in the reversal behaviors of worms treated with different dilutions of DNQX; therefore, a high drug concentration was used to ensure drug penetration. Two hours before training, $500 \mu \mathrm{l}$ of either $10 \mathrm{~mm}$ DNQX or $35 \mathrm{~mm} \mathrm{NaOH}$ (vehicle) was placed onto the surface of agar plates bearing E. coli. For the drug groups, the $500 \mu \mathrm{l}$ of $10 \mathrm{~mm}$ DNQX applied to $10 \mathrm{ml}$ of agar made the effective external concentration in the worm's environment $476 \mu \mathrm{m}$. One hour before training, four groups of 10-15 4-d-old wild-type worms were transferred to drug-treated plates. Groups of worms were given either taps in the distributed training protocol at a $60 \mathrm{sec}$ ISI or the single-tap control and then $1 \mathrm{hr}$ later were transferred to individual drugfree plates for testing with 10 taps (at a $60 \mathrm{sec}$ ISI) the next day. Drug exposure was $<6 \mathrm{hr}$, and all testing was conducted $\geq 20 \mathrm{hr}$ after removal of drug.

All behavioral data were scored using stop-frame video analysis to trace the distance traveled backward to each tap or train onto acetate sheets. Tracings were scanned into the computer and measured using NIH Image software. Occasionally, worms swam rapidly forward in response to tap; these accelerations forward are qualitatively different from reversals and were scored as missing data points ( $<10 \%$ of the data).

Imaging procedures. For imaging, GFP transgenic worm strains were mounted onto welled slides using $12 \mu \mathrm{l}$ of 2,3-butanedione monoxime for paralysis mixed with medium Sephadex beads to prevent worms from being crushed. A Nikon (Tokyo, Japan) Optiphot-2 microscope with an MRC 600 confocal system (Bio-Rad, Hercules, CA) equipped with a krypton-argon laser was used for imaging. GFP was excited using a 488 $\mathrm{nm}$ wavelength laser setting with emitted light collected passing through a 510-550 nm bandpass filter. Images were captured in a $768 \times 512$ pixel field of view, with optical sections collected at $0.5 \mu \mathrm{m}$ intervals using a $60 \times$ oil lens.

Twenty-four hours before imaging, GLR-1::GFP and ${ }_{\mathrm{p}}$ mec-7::SNB$1::$ GFP worms were given taps in the distributed training protocol or the single-tap control. One hour after training, worms were transferred to individual plates for imaging $24 \mathrm{hr}$ later.

The GLR-1::GFP strain expressed GFP along the ventral cord, and images were collected along the posterior portion from the tail to the vulva. Images of GLR-1::GFP expression were composed of 10-15 optical sections. A researcher blind to the treatment groups measured images. Collected projection images were viewed and measured using NIH Image 1.61. GFP expression was measured as the length of GFP clusters because ventral cord width was relatively uniform across animals.

GFP expressed in the ${ }_{\mathrm{p}}$ mec-7::SNB-1::GFP worms was captured in a single image composed of $\sim 12-18$ optical sections. Because the GFP in these worms was often faint, the microscope was set to maximal sensitivity for all worms. A researcher blind to the treatment groups measured images. In NIH Image, a threshold adjustment produced high-contrast images in black and white to allow for viewing of faint GFP. Area measurements for each region of GFP expression were calculated by outlining the GFP-expressing region and using the area measure function. Final figures were generated using Adobe Photoshop 7.0 (Adobe Systems, San Jose, CA).

\section{Results}

\section{Behavioral analyses of $g l r-1$ in long-term memory}

Both of the $g l r-1$ alleles were originally hypothesized to lead to null mutations (Hart et al., 1995; Maricq et al., 1995); however, our data showing differences in response magnitude to different stimuli suggests that the truncated protein produced by $k y 176$ is not totally lacking in function. $k y 176$ showed almost no reversal to tap, whereas $n 2461$ showed reversal responses to tap similar to wild-type worms (response to tap in pixels: $n=20 \mathrm{glr}-1(n 2461)$ worms, 220.03 $\pm 49.18 ; n=10 \mathrm{glr}-1$ ( $k y 176)$ worms, $5.95 \pm 4.04)$. Both strains reversed in response to a stronger stimulus, a train of taps (response to train: $n=19 \mathrm{glr}-1(n 2461)$ worms, $744.64 \pm$ $50.59 ; n=16 \operatorname{glr}-1$ (ky176) worms, $542.88 \pm 65.00)$. Therefore, for assessing both short- and long-term habituation, $g l r-1$ (n2461) was tested with both trains and taps, whereas $g l r-1(k y 176)$ was only tested with trains.

Despite differences in initial response magnitude, both $g l r-1$ strains showed significant short-term habituation to 20 stimuli at a $60 \mathrm{sec}$ ISI (Fig. 1), suggesting that the gene does not play a direct role in short-term habituation. To examine the role of $g l r-1$ in LTM for habituation, we used a between-groups distributed 
a

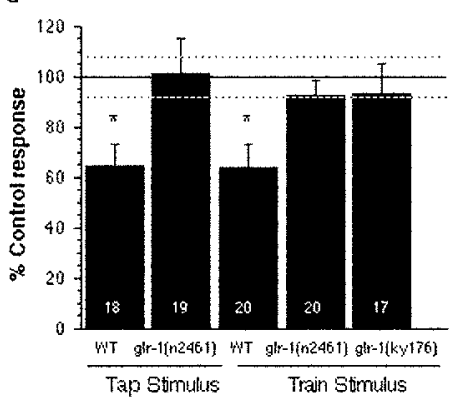

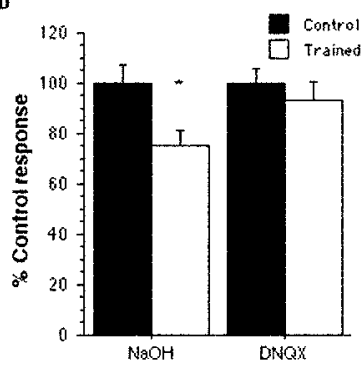
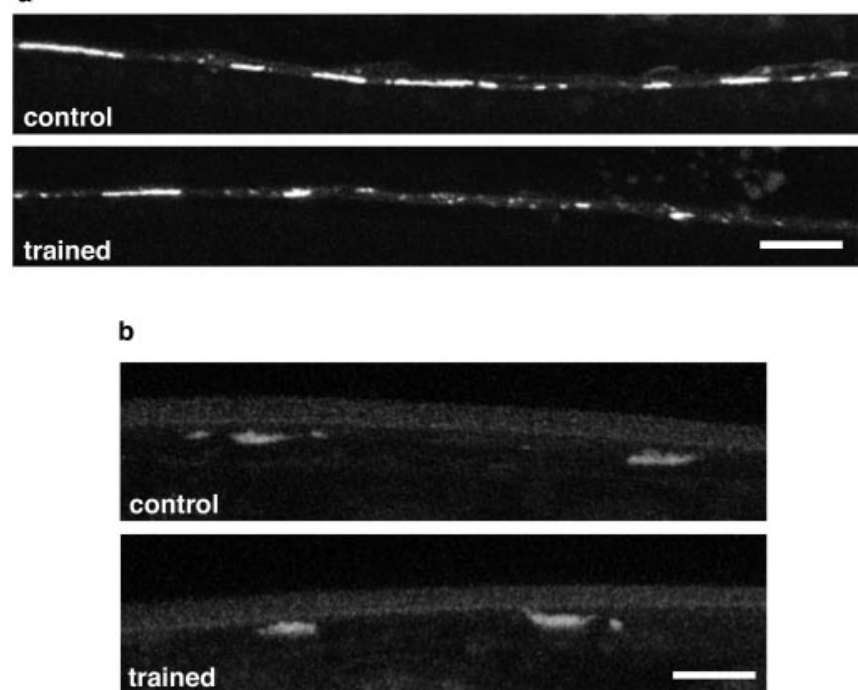

Figure 2. The role of $g / r-1$ glutamate receptors in memory. $a$, Mutations in glr- 1 (results from 2 alleles, $n 2461$ and $k y 176$ ) eliminate long-term memory for habituation. Mean percentage of control response magnitude for LTM for habituation training of the tap withdrawal response in wild-type (WT) and $g / r-1(n 2461)$ and $g / r-1(k y 176)$. Average reversal magnitude across the test trial for trained groups (black bars) expressed as percentage of control response (top black line across graph; dotted lines represent average \pm SEM for control groups). White numbers in bars represent the number of worms in each trained group. Both wild-type (WT) groups showed significant LTM; none of the glr- 1 groups showed significant LTM to either taps or trains. $b$, Exposure to the non-NMDA glutamate receptor antagonist DNQX during training blocks LTM for habituation of the tap withdrawal response in wild-type worms, and exposure to the vehicle control $\mathrm{NaOH}$ during training does not affect formation of LTM.

training protocol (training, four blocks of 20 stimuli at a $60 \mathrm{sec}$ ISI each separated by $1 \mathrm{hr}$ rest periods; testing, 10 stimuli at a $60 \mathrm{sec}$ ISI $24 \mathrm{hr}$ after training) to test wild-type N2, n2461, and ky176 worms. On day 2, when we compared the test responses of trained wild-type worms to trained $g l r-1$ mutant worms, we found that N2 worms showed LTM for both trains and taps, and neither $g l r-1$ allele showed LTM for either taps or trains (Fig. 2a). An ANOVA comparing the trained and control groups was significant $\left(F_{(5,108)}=3.17 ; p=0.01\right)$. PLSD post hoc comparisons showed that wild-type worms showed LTM in the form of significantly lower responses than control when trained with both taps $(p=0.01)$ and trains $(p=0.01)$; none of the $g l r-1$ trained groups differed significantly from control $(p>0.05$ in all cases). In addition, the responses of $g l r-1$ (n2461) trained worms were significantly larger than the responses of trained wild-type worms with both taps $(p=0.009)$ and trains $(p=0.03)$, and the responses of $g l r-1(k y 176)$ trained worms were significantly larger than the responses of trained wild-type worms with trains ( $p=$ $0.04)$.

To confirm the importance of $g l r-1$ receptors in LTM, N2 worms were treated with the competitive non-NMDA glutamate receptor antagonist DNQX (Sigma, St. Louis, MO). DNQX blocks neurotransmission at the Aplysia sensory neuron to motor neurons synapse (Dale and Kandel, 1993; Chitwood et al., 2001). DNQX does not block short-term homosynaptic depression (the cellular analog of habituation) (Armitage and Siegelbaum, 1998) but does block lasting memory for habituation in Aplysia (Ezzeddine and Glanzman, 2002). In C. elegans, neither DNQX nor the vehicle control $\mathrm{NaOH}$ blocked significant short-term habituation to 20 taps at a $60 \mathrm{sec}$ ISI $(t$ test for mean of responses 1 and 2 vs mean of responses 19 and $20: \mathrm{NaOH}, t_{(11)}=3.2, p=0.008$; DNQX, $\left.t_{(11)}=3.3, p=0.007\right)$. N2 worms were given distributed training on agar plates treated with $35 \mathrm{~mm} \mathrm{NaOH}$ (vehicle; $n=$ 22) (Fig. $2 b)$ or $10 \mathrm{~mm}$ DNQX $(n=27)$ dissolved in $\mathrm{NaOH}$. Matched control groups ( $n=21$ for $\mathrm{NaOH} ; n=24$ for DNQX) underwent the same drug treatments but received only one tap during the training phase. An ANOVA comparing trained DNQX and trained $\mathrm{NaOH}$ worms against untrained control worms was significant $\left(F_{(2,69)}=4.1 ; p=0.02\right)$. PLSD post hoc

Figure 3. Confocal images of GFP expression in trained and control worms. $a$, Confocal images of postsynaptic expression of $g / r-1$ receptors on processes of the interneurons in the posterior ventral nerve cord visualized with GLR-1::GFPshowing a single-tap control worm (control) and a worm that received distributed habituation training $24 \mathrm{hr}$ earlier (trained; same procedure as Fig. 2). The training produced behavioral LTM in another group of these transgenic worms $\left(t_{(44)}=2.5 ; p=0.02\right.$; data not shown). There were significantly smaller GFP clusters in the trained worms $(x=280.95 \pm 32.37 ; n=21)$ than in the single-stimulus control worms $\left(x=463.70 \pm 43.74 ; n=16 ; t_{(35)}=3.44 ; p=0.002\right)$, although the number of clusters between groups did not differ (trained, $62.2 \pm 7.14$; control, $69.3 \pm 10.3 ; t_{(35)}=0.59 ; p=$ 0.56). Scale bar, $20 \mu \mathrm{m} . b$, Confocal images of the vesicles in tap sensory neuron terminals visualized with a synaptobrevin GFP marker showing a single-tap control worm (control) and a worm that received distributed habituation training $24 \mathrm{hr}$ earlier (trained). The training produced behavioral LTM in another group of these transgenic worms $\left(t_{(44)}=2.45 ; p=0.02\right.$; data not shown). There was no difference in measured GFP expression between the trained worms $(x=41.00 \pm 3.47 ; n=12)$ and the single-stimulus control worms $(x=42.5 \pm 5.4 ; n=12$; $\left.t_{(22)}=0.41 ; p=0.69\right)$. Scale bar, $20 \mu \mathrm{m}$.

tests showed significant differences between $\mathrm{NaOH}$ trained and control worms $(p=0.007)$ and between $\mathrm{NaOH}$ trained and DNQX trained worms $(p=0.04)$; there was no significant difference between DNQX trained worms and control worms ( $p=$ $0.45)$. Thus, exposure to vehicle during training had no effect on expression of LTM, and exposure to vehicle plus DNQX during training blocked the development of LTM for habituation.

\section{Training alters GLR-1::GFP expression}

Together, the experiments with the $g l r-1$ worms and the wildtype worms exposed to DNQX show that the $g l r-1$ receptor is critical for LTM of habituation in the tap withdrawal circuit. This suggested that habituation training might alter $g l r-1$ expression. To investigate this possibility, we tested worms carrying chimeric receptors made up of GLR-1 tagged with green fluorescent protein (GLR-1::GFP) to visualize changes in $g l r-1$ expression (Rongo and Kaplan, 1999). In GLR-1::GFP worms, fluorescence is localized to clusters along the ventral cord neurites and in the nerve ring (corresponding to anatomical synaptic markers reported in EM studies) (White et al., 1986). Confocal imaging was used to visualize the GLR-1::GFP. We compared the amount of GLR-1::GFP expression in the ventral cord in trained worms and control worms. Worms that received distributed training $24 \mathrm{hr}$ earlier showed no difference in the number of GLR-1::GFP clusters in the ventral cord; however, the overall length of GFP clusters per worm in the trained worms was significantly shorter than in the control worms (Fig. 3a). These data led us to hypothesize 
that long-term habituation training did not produce a change in the number of synapses but did reduce the number of $g l r-1$ receptors expressed per synapse on the interneurons.

To look for the presynaptic changes after training, we measured GFP expression of the gene for C. elegans synaptobrevin $(s n b-1)$, a protein associated with synaptic vesicles, in the tap sensory neurons. SNB-1::GFP was expressed under the control of the mec-7 promoter that restricts GFP expression to the six mechanosensory neurons of the tap circuit (Nonet, 1999). In these worms, we focused on an accumulation of fluorescence in areas that corresponded with a specific set of tail-touch cell synaptic connections seen in EM studies (White et al., 1986). The fluorescence we measured appeared as one or two patches in the ventral cord just posterior to the vulva (Nonet, 1999). We measured the total area of the patches in the ventral cords of worms that received distributed training and control worms and found that there was no difference in the amount of SNB-1::GFP expression in the two groups (Fig. 3b). Thus, LTM for habituation did not affect the expression of synaptobrevin in these sensory neuron terminals.

Because the behavioral expression of LTM for habituation can be blocked by exposure to heat shock in the $1 \mathrm{hr}$ rest intervals between training blocks (Beck and Rankin, 1977; Rose et al., 2002), we tested whether the heat shock would alter the effect of training on the expression of GLR-1::GFP. We found that, $24 \mathrm{hr}$ after training, the mean length of GLR-1::GFP clusters in worms that received heat shock $\left(32^{\circ} \mathrm{C}\right.$ for 40 min after each training block) during distributed habituation training on day 1 did not differ from worms that received only a single tap on day 1 (trained with heat shock, $n=18, x=496.95 \pm 50.2$; control with heat shock, $n=16, x=468.75 \pm 54.26$; $\left.t_{(31)}=0.380 ; p=0.71\right)$. In contrast, worms that received distributed training with sham heat shock $\left(21^{\circ} \mathrm{C}\right.$ for $40 \mathrm{~min}$ after each training block) showed decreased mean length of GLR-1::GFP clusters $24 \mathrm{hr}$ after training when compared with their single-tap controls (trained with sham shock, $n=18, x=374.8 \pm 26.38$; control with sham shock, $n=$ $\left.18, x=485.26 \pm 33.47 ; t_{(34)}=2.6 ; p=0.01\right)$. Thus, heat shock blocked the alteration in GLR-1::GFP expression usually seen after distributed habituation training, without affecting any other aspect of GLR-1::GFP expression (compare GLR-1::GFP levels in control heat shock and sham shock groups).

\section{Discussion}

In behavioral studies, we showed that mutations in the excitatory glutamate receptor gene $g l r-1$ did not affect short-term memory for habituation in C. elegans but blocked LTM. A glutamate receptor antagonist also blocked LTM for habituation. Therefore, the receptor encoded by the $g l r-1$ gene is hypothesized to play a critical role in LTM for habituation in the tap withdrawal response in C. elegans. Interestingly, in the rat, bilateral infusion of an AMPA-receptor antagonist immediately after training impaired LTM for habituation to a novel environment (Vianna et al., 2000).

When we used confocal imaging to examine expression of GLR-1::GFP $24 \mathrm{hr}$ after LTM training, we found no decrease in the number of GFP clusters observed but a significant decrease in the overall amount of GFP expressed. This suggested that, although there was no change in the number of synapses, there was a change in the density of receptors at the synapses. The observation that there was no change in the amount of synaptobrevin in the terminals of the sensory neurons suggests that training and the decrease of postsynaptic glutamate receptors did not alter the number of vesicles in the presynaptic terminal. This was an un- expected result because, in Aplysia, long-term memory for habituation is correlated with a presynaptic decrease in the number of synapses, size of synapses, and number of vesicles in active zones; no studies have looked postsynaptically (Bailey and Chen, 1983). One possible explanation for the differences is that, in our experiments, we used only a short training regimen that produces behavioral LTM; the Aplysia received extensive training, much more than would be needed to produce behavioral changes. We recently collected pilot data that suggests that, if worms are overtrained, there are decreases in the number of vesicles in the sensory neuron terminals.

The observation that heat shock blocks the behavioral expression of LTM after distributed but not massed training has been used to support the hypothesis that the LTM observed at $24 \mathrm{hr}$ after training is protein synthesis dependent. Heat shock is thought to stop ongoing protein synthesis and block new protein synthesis from occurring for a critical period of time after each training block (Beck and Rankin, 1975; Rose et al., 2002). The finding that heat shock also blocks the change in GLR-1::GFP expression suggests that this too is protein synthesis dependent.

This is the first study to simultaneously examine both presynaptic and postsynaptic anatomical changes in the nervous system of living animals induced using the same stimulus protocol that produces behavioral expression of long-term memory. The data from these experiments suggest the hypothesis that long-term memory for habituation in C. elegans is mediated not by a change in the number of synapses but by a downregulation of nonNMDA-type glutamate receptors on the postsynaptic interneurons that weakens the strength of the sensory to interneuron synapses. This is consistent with data on AMPA receptor trafficking that is being reported in mammalian LTD preparations in which electrical stimulation or drug treatments that produce a weakening of synaptic strength also produce a downregulation of surface-expressed AMPA receptors (for review, see Bear and Linden, 2001; Lüscher and Frerking, 2001; Malinow and Malenka, 2002). Heynen et al. (2000) have suggested that memory is encoded by experience-dependent assignment of glutamate receptors to synapses. C. elegans offers an exciting in vivo system to study glutamate receptor trafficking as it relates to behavioral changes and experience.

\section{References}

Armitage BA, Siegelbaum SA (1998) Presynaptic induction and expression of homosynaptic depression at Aplysia sensorimotor neuron synapses. J Neurosci 18:8770-8779.

Avery L (1993) The genetics of feeding in Caenorhabditis elegans. Genetics 133:897-917.

Bailey CH, Chen M (1983) Morphological basis of long-term habituation and sensitization in Aplysia. Science 220:91-93.

Bear MF (2001) The mechanisms and meaning of long-term synaptic depression in the mammalian brain. In: Synapses (Cowan WM, Sudhof TC, Stevens CF, eds), pp 455-517. Baltimore: Johns Hopkins UP.

Beck CDO, Rankin CH (1995) Heat-shock disrupts long-term memory consolidation in Caenorhabditis elegans. Learn Mem 2:161-177.

Beck CDO, Rankin CH (1997) Long-term habituation is produced by distributed training at long ISIs and not by massed training or short ISIs in Caenorhabditis elegans. Anim Learn Behav 25:446-457.

Bellocchio EE, Hu H, Pohorille A, Chan J, Pickel VM, Edwards RH (1998) The localization of the brain-specific inorganic phosphate transporter suggests a specific presynaptic role in glutamatergic transmission. J Neurosci 18:8648-8659.

Brenner S (1974) The genetics of Caenorhabditis elegans. Genetics 77:71-94. Chalfie M, Sulston JE, White JG, Southgate E, Thomson JN, Brenner S (1985) The neural circuit for touch sensitivity in Caenorhabditis elegans. J Neurosci 5:956-964 .

Chitwood RA, Li Q, Glanzman DL (2001) Serotonin facilitates AMPA-type 
responses in isolated siphon motor neurons of Aplysia in culture. J Physiol (Lond) 534:501-510.

Dale N, Kandel E (1993) L-glutamate may be the fast excitatory transmitter of Aplysia sensory neurons. Proc Natl Acad Sci USA 90:7163-7167.

Ezzeddine YM, Glanzman DL (2002) Role of protein synthesis and protein kinase activity in long-term habituation of the gill-withdrawal reflex in Aplysia. Soc Neurosci Abstr 28:376.6.

Hart AC, Sims S, Kaplan JM (1995) Synaptic code for sensory modalities revealed by C. elegans GLR-1 glutamate receptor. Nature 378:82-85.

Heynen AJ, Quinlan EM, Bae DC, Bear MF (2000) Bidirectional, activitydependent regulation of glutamate receptors in the adult hippocampus in vivo. Neuron 28:527-536.

Lee RY, Sawin ER, Chalfie M, Horvitz HR, Avery L (1999) EAT-4, a homolog of a mammalian sodium-dependent inorganic phosphate cotransporter, is necessary for glutamatergic neurotransmission in Caenorhabditis elegans. J Neurosci 19:159-167.

Lüscher C, Frerking M (2001) Restless AMPA receptors: implications for synaptic transmission and plasticity. Trends Neurosci 24:665-670.

Malinow R, Malenka RC (2002) AMPA receptor trafficking and synaptic plasticity. Annu Rev Neurosci 25:103-126.

Maricq AV, Peckol E, Driscoll M, Bargmann CI (1995) Mechanosensory signalling in C. elegans mediated by the GLR-1 glutamate receptor. Nature 378:78-81.

Nonet ML (1999) Visualization of synaptic specializations in live C. elegans with synaptic vesicle protein-GFP fusions. J Neurosci Methods 89:33-40.

Rongo C, Kaplan JM (1999) CaMKII regulates the density of central glutamatergic synapses in vivo. Nature 402:195-199.

Rose JK, Chen S, Kaun KR, Rankin CH (2001) Glutamate and AMPAreceptor function are necessary for long-term memory in C. elegans. Soc Neurosci Abstr 27:87.9.

Rose JK, Kaun KR, Rankin CH (2002) A new group-training procedure for habituation demonstrates that presynaptic glutamate release contributes to long-term memory in C. elegans. Learn Mem 9:130-137.

Vianna MR, Alonso M, Viola H, Quevedo J, de Paris F, Furman M, de Stein ML, Medina JH, Izquierdo I (2000) Role of hippocampal signaling pathways in long-term memory formation of a nonassociative learning task in the rat. Learn Mem 7:333-340.

White JE, Southgate E, Thomson JN, Brenner S (1986) The structure of the nervous system of the nematode Caenorhabditis elegans. Philos Trans R Soc Lond B Biol Sci 314:1-340.

Wicks SR, Rankin CH (1995) Integration of mechanosensory stimuli in Caenorhabditis elegans. J Neurosci 15:2434-2444. 